\title{
Effectiveness of Highly Active Antiretroviral Therapy (HAART) Used Concomitantly With Rifampicin in Patients with Tuberculosis and AIDS
}

\author{
Flávia Marinho Sant’Anna ${ }^{1}$, Luciane Velasque ${ }^{1}$, Marli Jane Costa ${ }^{1}$, Carolina Arana Schmaltz ${ }^{1}$, Mariza Gonçalves Morgado ${ }^{2}$, \\ Maria Cristina Lourenço ${ }^{3}$, Beatriz Grinsztejn ${ }^{1}$ and Valéria Cavalcanti Rolla ${ }^{1}$ \\ ${ }^{1}$ Instituto de Pesquisa Clínica Evandro Chagas, Fundação Oswaldo Cruz; ${ }^{2}$ Laboratório de AIDS \& Imunologia Molecular, Instituto Oswaldo \\ Cruz, Departamento de Imunologia, Fundação Oswaldo Cruz; ${ }^{3}$ Laboratório de Bacteriologia, Instituto de Pesquisa Clínica Evandro Chagas, \\ Fundação Oswaldo Cruz; Rio de Janeiro, RJ, Brazil
}

\begin{abstract}
This study evaluated the effectiveness of two HAART regimens concomitant to rifampicin based tuberculosis (TB) treatment. Patients with TB/HIV diagnosis followed at the TB program between June 2000 and March 2005 were prospectively evaluated. The different HAART regimens in antiretrovirals (ARV) treatment naïve and ARV experienced patients were compared. The effectiveness of HAART was defined as a VL $<80$ copies/mL from month 4 to month 10 after TB treatment. One hundred and forty-two patients were included. Among these, 68 (47\%) were treatment naïve and $76(53 \%)$ previously exposed. Odds ratio (OR) in naïve patients treated with efavirenz (EFV) based regimen $(n=42)$ compared to ritonavir/saquinavir $(\mathrm{RTV} / \mathrm{SQV})$ based regimen $(\mathrm{n}=26)$ was $8.0(\mathrm{CI}=1.67-38.35, \mathrm{p}=0.008)$. OR from ARV experienced patients treated with RTV/SQV based regimen compared to EFV was 3.08 (CI=0.65-14.6, $p=0.15)$, although with no statistical significance. Better effectiveness and tolerability were observed in antiretrovirals treatment naïve patients using EFV based regimens. Although not statistically significant, a favorable virologic response and a better tolerability were observed in the ARV experienced patients group who received a RTV/SQV based regimen.
\end{abstract}

Key-Words: Tuberculosis, AIDS, HAART, rifampicin, effectiveness.

Globally, the incidence of opportunistic infections among AIDS patients has declined after HAART introduction. However, tuberculosis (TB), among other infectious diseases remains a major cause of morbidity in developing countries [1,2].

Antiretroviral treatment in HIV-TB patients represents a challenge due to the interactions of rifampicin with antiretroviral drugs [3]. Moreover, anti-TB treatment without rifampicin is longer (1 year), uncomfortable, and more frequently associated with tuberculosis reappearance [4]. Rifampicin interacts with protease inhibitors (PI) and with non-nucleosides reverse transcriptase inhibitors (NNRTI), inducing cytochrome P450 and decreasing PI and NNRTI plasma levels [5,6]. Side effects and drug-drug interactions must be considered when concurrent treatment of HIV and tuberculosis is administrated.

Factors of great relevance, as HAART effectiveness and comparative tolerability of antiretrovirals use concomitant to rifampicin, were poorly evaluated. Studies conducted so far have evaluated only one regimen and used small series $[3,7,9]$. The aim of this study was to evaluate the effectiveness of HAART regimens recommended in the Brazilian Guidelines, concomitant to rifampicin based tuberculosis treatment.

\section{Material and Methods}

We conducted a prospective study in a cohort of HIVpositive patients with tuberculosis (TB) diagnosis, followed

Received on 15 May 2009; revised 8 September 2009.

Address for correspondence: Dr. Valéria Cavalcanti Rolla. Instituto de Pesquisa Clínica Evandro Chagas, Fundação Oswaldo Cruz. Avenida Brasil, 4365 - Manguinhos. Zip code: 21045-900 - Rio de Janeiro, Brazil. Phone +55 21 38659601. Fax: + 5521 38659601. E-mail: valeria.rolla@ipec.fiocruz.br.

The Brazilian Journal of Infectious Diseases 2009;13(5):362-366. (C) 2009 by The Brazilian Journal of Infectious Diseases and Contexto Publishing. All rights reserved. at the Tuberculosis Clinic of the Instituto de Pesquisa Clínica Evandro Chagas (IPEC), Fundação Oswaldo Cruz, Rio de Janeiro, Brazil, from July 2000 to March 2005.

Patients with 18 years or older, HIV-positive, with signs and/or symptoms suggestive of tuberculosis (TB), confirmed by positive culture or a successful therapeutic test, defined as clinical and radiological improvement after the exclusion of other potential opportunistic diseases that might explain the condition, were included. Antiretroviral (ARV) naïve and patients already under ARV therapy were eligible.

Patients with TB diagnosis and no indication to initiate antiretroviral treatment, individuals that for some reason did not use rifampicin based tuberculosis treatment, or that for any reason did not perform viral load (VL) tests after TB treatment were excluded. Follow-up visits were performed at $15,30,60,120$, and 180 days after initiation of tuberculosis treatment. For the ARV naïve patients, HAART was initiated at least 30 days after the tuberculosis regimen was started. CD4 count was performed approximately 15 days after initiation of the TB treatment to evaluate indication of ARV. HIV plasma VL was performed 4 to 10 months after the TB therapy initiation. For those patients already on ARV at the moment of TB diagnosis, CD4 and VL results within 90 days of the TB diagnosis were considered. Effectiveness of HAART was defined in this study as a VL $<80$ copies/mL obtained within the $4^{\text {th }}$ and the $10^{\text {th }}$ month post-TB treatment. Tuberculosis and HIV therapies were prescribed according to the Brazilian Ministry of Health recommendations [8], i.e., rifampicin 600mg, isoniazid 400mg, pyrazinamide $2000 \mathrm{mg}$ for 2 months, followed by rifampicin 600mg and isoniazid 400mg during 4 more months. Ethambutol 1200mg was added for patients with a medical history of previous tuberculosis treatment. Dose adjustment of the drugs was performed according to body weight, when required [8]. 
The antiretroviral regimens used included two nucleoside analogs associated with efavirenz $600 \mathrm{mg}$ or ritonavir $400 \mathrm{mg} /$ saquinavir 400mg. HAART regimens were prescribed according to Brazilian Ministry of Health guidelines. Safety was accessed by the presence of severe (grade III) or potentially life threatening (grade IV) adverse events that led to HAART interruption during TB treatment.

Logistic regression model was applied to evaluate the impact of covariates in the outcome (VL $<80$ copies/mL). Univariate analysis was performed for each covariate, including: median age ( $\leq 36$ years vs. $>36$ years), gender, clinical forms of TB (disseminated vs. pulmonary/extrapulmonary) CD4 count in TB treatment initiation (<200 vs. $\geq 200$ ), exposition category (heterosexual, homosexual/ bisexual, other) viral load in TB treatment initiation $(\leq 80$ copies/mL vs. $>80$ copies/mL) and ARV naïve (Yes vs. No). Covariates reaching up to $25 \%$ significance $(\mathrm{p} \leq 0.25)$ in univariate analysis were included in the multivariate model. Multivariate analysis was stratified by ARV naïve as an indicator variable. The model followed the stepwise backward strategy, and the criterion for the exclusion of the variables was the highest p-value. Variables with clinical significance $(\mathrm{OR}>1.30)$ or statistical significance $(p<0.05)$ were kept in final model. The statistical analysis was performed using SAS software (SAS system version 9.2).

This project was approved by the "Comitê de Ética em Pesquisa” from the Instituto de Pesquisa Clínica Evandro Chagas, and all patients who accepted to participate in the study signed an informed consent form.

\section{Results}

From July 2000 to March 2005, one hundred forty two TB-AIDS patients were followed. Fifty-four (38\%) were women and $56 \%$ were white race. The mean of age was thirty-seven years and $62 \%$ of the patients were heterosexual. The most frequent clinical forms of TB were pulmonary (61 patients - 43\%), disseminated (44 patients $31 \%$ ) and ganglionar form (21 patients 15\%). Mean and median baseline CD4 count were 169 (SD=147) and 132 cells/ $\mathrm{mm}^{3}$ respectively. Mean and median baseline VL were 4.7 $\log (\mathrm{SD}=1.26$ and $5.1 \mathrm{log}$, respectively).

Sixty six (46.5\%) patients were antiretroviral treatment naïve and 76 (53\%) had previous experience to antiretrovirals. Demographic and clinical characteristics of patients are shown in Table 1.

During follow-up, 22 (15.5\%) patients died due to TB or AIDS. Twenty (13.8\%) patients did not perform a VL test within 4 and 10 months after TB treatment initiation (nine of them were lost of follow up).

A total of 100 patients were followed until the end of the study and included in the logistic regression analysis. All patients achieved the cure criteria for tuberculosis and 54\% patients achieved a VL $<80$ copies/mL within 4 and 10 months after TB treatment.
Antiretroviral Treatment Naïve Patients

Among the 53 treatment naïve patients, baseline mean and median CD4 counts at beginning of tuberculosis treatment were $150\left(\mathrm{SD}=145\right.$ and 107 cells/ $\mathrm{mm}^{3}$, respectively and mean $\mathrm{VL}$ was $\mathrm{f} 5.2 \log (\mathrm{SD}=0.9)$. More than half of these patients (53\%) were treated with an efavirenz based regimen, during all TB treatment. Among the 25 (47\%) patients who initiated a ritonavir/saquinavir based regimen, only 11 (20\%) remained on this regimen until the end of the TB treatment. Moreover, the fourteen patients who discontinued this regimen did it due to severe HAART treatment related adverse events. These patients were switched to an efavirenz based regimen, with good tolerability in all cases. Among patients treated with an efavirenz based regimen 64\% (18/28) achieved VL $<80$ copies. Among those treated with a ritonavir/saquinavir regimen $27 \%$ (3/11) achieved VL $<80$ copies and 57\% (8/14) of those who switched therapy achieved a $\mathrm{VL}<80$ copies.

\section{ARV Experienced Patients}

Among the 47 ARV experienced patients mean and median baseline CD4 counts at the beginning of tuberculosis treatment were 191.8 cells $(\mathrm{SD}=149)$ and 160 cells $/ \mathrm{mm}^{3}$, respectively. Mean and median VL were $4.2 \log (\mathrm{SD}=1.4)$ and $4.5 \mathrm{log}$, respectively. Eight patients (16\%) used a saquinavir/ ritonavir based regimen and 58 (41\%) an efavirenz based regimen.

ARV experienced patients who were treated with efavirenz during TB therapy received multiple regimens before TB diagnosis. Most included an efavirenz based regimen $(\mathrm{N}=29)$ and some a PI based regimen (the most frequent was lopinavir/ $r, \mathrm{~N}=2)$. Other regimens used were: indinavir/r $(\mathrm{N}=3)$, and nelfinavir $(\mathrm{N}=2)$.

Among patients treated with an efavirenz based regimen, 48\% (19/39) achieved VL <80 copies; for those treated with a ritonavir/saquinavir based regimen, 75\% (6/8) achieved $\mathrm{VL}<80$ copies. In this group of ARV experienced patients, both treatments (TB and HIV) were well tolerated, and no serious adverse events leading to treatment change occurred.

ARV experienced patients who were treated with ritonavir/ saquinavir during TB therapy received different regimens before TB diagnosis, that included: double nucleoside analogs with AZT and 3TC $(\mathrm{N}=4)$, indinavir/r based regimen $(\mathrm{N}=4)$, not boosted indinavir $(\mathrm{N}=1)$, and nelfinavir based regimen $(\mathrm{N}=3)$.

Univariate and Multivariate Analysis

In the univariate analysis, race, age, TB clinical presentation, and CD4 cell count at TB diagnosis did not show an association with an undetectable viral load (VL $<80$ copies), as shows in Table 2.

Factors that entered the initial multivariate logistic model included HAART, exposure category, ARV naïve and viral load at the beginning of TB treatment.

In the adjusted model by HAART, exposure category, clinical presentation of TB and viral load at beginning of TB, treatment naïve patients had 2.95 times higher chance to 
Table 1. Demographic, epidemiologic and clinical data from 142 patients with AIDS and tuberculosis, followed at IPEC/FIOCRUZ, from 2000-2005.

\begin{tabular}{|c|c|}
\hline Variable & $\mathbf{N}(\%)^{*}$ \\
\hline \multicolumn{2}{|l|}{ Gender } \\
\hline Women & $54(37)$ \\
\hline Men & $90(63)$ \\
\hline \multicolumn{2}{|l|}{ Race } \\
\hline White & $78(55)$ \\
\hline Black & $64(45)$ \\
\hline \multicolumn{2}{|l|}{ Age (years) } \\
\hline $18-30$ & $38(27)$ \\
\hline $31-49$ & $90(63)$ \\
\hline$\geq 50$ & $14(10)$ \\
\hline Mean (SD*) & 37 (8) \\
\hline Median & 37 \\
\hline \multicolumn{2}{|l|}{ Exposure category } \\
\hline Heterosexual & $89(63)$ \\
\hline Homosexual / bisexual & $49(34)$ \\
\hline Other & $4(3)$ \\
\hline \multicolumn{2}{|l|}{ ARV naïve } \\
\hline Yes & $66(47)$ \\
\hline No & 76 (53) \\
\hline \multicolumn{2}{|l|}{ Clinical presentation of TB } \\
\hline Disseminated & $30(21)$ \\
\hline Pulmonary/Extra-pulmonary & $112(79)$ \\
\hline \multicolumn{2}{|c|}{ HAART regimen during TB treatment ${ }^{* *}$} \\
\hline RTV-SQV & $23(16)$ \\
\hline EFV & $89(63)$ \\
\hline RTV/SQV changed to EFV & 15 (11) \\
\hline \multicolumn{2}{|c|}{ CD4 count at the beginning of TB treatment } \\
\hline$\leq 200$ cells $/ \mathrm{mm}^{3}$ & $84(59)$ \\
\hline$>200$ cells $/ \mathrm{mm}^{3}$ & $58(34)$ \\
\hline \multicolumn{2}{|c|}{ Viral load at the beginning of $\mathrm{TB}$ treatment } \\
\hline$\leq 80$ copies $/ \mathrm{mL}$ & $12(10)$ \\
\hline$>80$ copies $/ \mathrm{mL}$ & $104(90)$ \\
\hline
\end{tabular}

achieve undetectable viral load at the end of TB treatment than ARV experienced patients ( $\mathrm{p}=0.008$, data not showed).

Further analysis was conducted separately for antiretroviral naïve and ARV experienced patients, as shows Table 3. In these models the variables "category of exposure and viral load at the beginning of TB" were not kept in the final model, as they did not show any effect, and have not reached statistical significance.

When a model including only treatment naïve patients was performed (model A), solely the variable "type of antiretroviral regimen" showed a significant association with the outcome. Patients who used efavirenz had a 7 times higher chance of achieving undetectable viral load (VL $<80$ copies) when compared to those using ritonavir/saquinavir all along. Patients who initiated treatment with ritonavir/saquinavir and switched to efavirenz had 4 times higher chance of achieving undetectable viral load when compared to those who remained on a ritonavir/saquinavir regimen (Table 3).
No co-variables showed a statistically significant association with the outcome in the model performed for the ARV experienced patients (model B). However, patients who used a ritonavir/saquinavir based regimen presented three times higher chances of having undetectable viral load at the end of the antituberculosis treatment, when compared with patients who used efavirenz (OR 3.08; 95\% CI 0.65-14.6) ( $\mathrm{p}=0.15$ ), (Table 3).

\section{Discussion}

Tuberculosis is a major cause of morbidity and mortality among HIV/AIDS patients. Early tuberculosis diagnosis in HIV infected individuals is crucial, in view of the immunodeficiency progression and the increase mortality associated to this disease $[10,13]$. Direct and indirect costs of both disease conditions are huge, primarily in developing countries, determining a strong impact on the economy [1]. 
Table 2. Univariate analysis for previously treated and antiretroviral naïve patients with AIDS and tuberculosis from 07/2000 to 03/2005.

\begin{tabular}{|c|c|c|}
\hline Univariate Model & OR (CI 95\%) & p-value \\
\hline \multicolumn{3}{|l|}{ ARV therapy } \\
\hline RTV-SQV & 1 & \\
\hline EFV & $2.45(1.04-5.75)$ & 0.03 \\
\hline Initiated RTV-SQV and changed & $3.93(1.12-13.83)$ & 0.03 \\
\hline \multicolumn{3}{|l|}{ Race } \\
\hline White & $1.10(0.55-2.17)$ & 0.78 \\
\hline Non white & 1 & \\
\hline \multicolumn{3}{|l|}{ Exposure category } \\
\hline Heterosexual & 1 & \\
\hline Homosexual/bisexual/Other & $1.72(0.86-3.84)$ & 0.12 \\
\hline \multicolumn{3}{|l|}{ Age (years) } \\
\hline$<37$ & $0.72(0.37-1.46)$ & 0.38 \\
\hline$\geq 37$ & 1 & \\
\hline \multicolumn{3}{|l|}{ ARV Naive } \\
\hline Yes & $1.51(0.77-2.98)$ & 0.22 \\
\hline No & 1 & \\
\hline \multicolumn{3}{|l|}{ TB clinical presentation } \\
\hline Disseminated & $1.14(0.50-2.60)$ & 0.75 \\
\hline Other (pulmonary and extrapulmonary) & 1 & \\
\hline \multicolumn{3}{|l|}{ Viral load at the beginning of TB treatment } \\
\hline$\leq 80$ copies $/ \mathrm{mL}$ & $2.06(0.61-6.94)$ & 0.24 \\
\hline$>80$ copies/mL & 1 & \\
\hline \multicolumn{3}{|c|}{$\mathrm{CD4}^{+}$cell count at the beginning of TB treatment } \\
\hline$\leq 200$ cells $/ \mathrm{mm}^{3}$ & $1.15(0.52-2.53)$ & 0.71 \\
\hline$>200$ cells $/ / \mathrm{mm}^{3}$ & 1 & \\
\hline
\end{tabular}

HAART was one of the greatest achievements of the last decade, as it reduces the HIV related morbidity and mortality both in developed and developing countries where this treatment is available $[14,15]$.

Our results show that the overall effectiveness of HAART among this cohort of HIV/TB co-infected patients was 54\%. When we evaluated the ritonavir/saquinavir based regimen in antiretroviral naïve patients, a lower proportion of effectiveness was observed (27\%) compared to an efavirenz based regimen (64\%) $(\mathrm{p}<0.04)$. Low tolerability to this dual PI based regimen was also seen, with $70 \%$ percent of these patients presenting severe or serious adverse events $[16,17]$ These events were mainly gastrointestinal, leading to the substitution of ritonavir/saquinavir for efavirenz, with good subsequent tolerability and virologic outcome (57\%). The addition of a dual PI based regimen on top of an already hepatotoxic regimen (antituberculosis) could potentially explain the low tolerability observed among our naïve patients [6]. Patients treated with an efavirenz based regimen presented better virologic and safety outcomes, suggesting that this drug is more effective for this patient population than a dual PI regimen. The best timing of HAART initiation during TB therapy for ARV naïve patients is still an unanswered question, and we cannot rule out its impact on our results. Recent results from the SAPIT (Starting Antiretroviral therapy in three Points In Tuberculosis therapy) trial, conducted in South Africa, showed a 56\% lower mortality with integrated TB/HIV treatment for patients with CD4 T-lymphocyte count less than 500 cells $/ \mathrm{mm}^{3}$. Randomized controlled trials of early versus delayed initiation of HAART for severely immunosuppressed patients are currently underway [18].

Efavirenz associated with rifampicin use was well tolerated in the ARV experienced patients group. We did not observe severe or serious adverse events requiring medication switch or hospitalization in any of the patients. However, the low effectiveness of this regimen, (only $48 \%$ of patients achieved an undetectable viral load) compared with the ritonavir/ saquinavir based regimen with a twofold chance of achieving a better virologic outcome ( $75 \%$ of patients, $\mathrm{p}=0.17$ ), points out the limitations of its use among this patient population. This limited effectiveness could be explained by the low genetic barrier of non nucleoside analogs [10] and the previous use of this drug among 29/47 of the patients. Although this is a well known limitation, this drug was chosen by the physician to allow the concomitant rifampicin use and to avoid the risk of the intolerance, and high pill burden of a dual PI regimen [11,12].

Despite ritonavir/saquinavir use has been associated with a higher discontinuation rate among antiretroviral naïve 
Table 3. Multivariate model for previous exposed and antiretroviral naïve patients with AIDS and tuberculosis from 07/2000 to 03/2005.

\begin{tabular}{|c|c|c|c|c|}
\hline \multirow[t]{2}{*}{ Variables } & \multicolumn{2}{|c|}{$\begin{array}{c}\text { Multivariate model } \\
\text { antiretroviral naïve (Model A) }\end{array}$} & \multicolumn{2}{|c|}{$\begin{array}{l}\text { Multivariate model ARV } \\
\text { experienced (Model B) }\end{array}$} \\
\hline & OR (95\% CI) & p-value & OR (95\% CI) & p-value \\
\hline \multicolumn{5}{|l|}{ Clinical presentation } \\
\hline Disseminated & $1.42(0.36-5.62)$ & 0.60 & $1.27(0.17-9.47)$ & 0.81 \\
\hline Pulmonary and extrapulmonary) & 1 & - & 1 & - \\
\hline \multicolumn{5}{|l|}{ ARV regimen* } \\
\hline RTV-SQV & 1. & - & $3.08(0.65-14.6)$ & 0.15 \\
\hline EFV & $8.0(1.67-38.35)$ & 0.009 & 1. & - \\
\hline Initiated RTV-SQV and switched & $4.90(0.92-26.17)$ & 0.06 & - & - \\
\hline \multicolumn{5}{|c|}{ CD4 count at the beginning of TB treatment } \\
\hline$<200$ cells $/ \mathrm{mm}^{3}$ & $1.67(0.47-6.03)$ & 0.42 & $1.06(1.50-2.23)$ & 0.87 \\
\hline$\geq 200$ cells $/ \mathrm{mm}^{3}$ & 1 & - & - & - \\
\hline
\end{tabular}

RTV-SQV= ritonavir/saquinavir; $\mathrm{EFV}=$ efavirenz; $\mathrm{ARV}=$ antiretroviral;TB= tuberculosis.

patients, no severe or serious adverse events were observed in ARV experienced patients group. These findings indicate that this combination has its applicability and may be a good option for this subset of patients. As they have less remaining treatment options, they may be more prone to tolerate a more complicated HAART regimen.

Overall HAART outcomes among patients on a rifampicin based antituberculosis treatment showed to be acceptable considering the complexity of TB-AIDS concomitant therapy. Adverse events were frequent among antiretroviral naive patients using a booster PI regimen but were absent among antiretroviral experienced patients. Among the antiretroviral naïve subset of patients, an efavirenz $600 \mathrm{mg}$ based regimen showed better virologic and safety outcomes. Although not statistically significant, favorable virologic and safety outcomes were observed among ARV experienced patients who used ritonavir/saquinavir 400/400mg regimen when compared to antiretroviral naïve patients.

\section{Acknowledgments}

We thank to CNPq (Millenio II - REDE TB) and PDTSP-SUS (Fiocruz) for the financial support, to Dr. Carla Vorsatz for the English revision and to all patients who participated in this study.

\section{References}

1. World Health Organization. Global tuberculosis control: Surveillence, planning, financing. World Health Organization Report 2008 [on the internet]. Geneva: WHO Press. Available at: http://www.who.int/tb/publications/global_report/2008.

2. Corbett E.l., Watt C.J., Walker N. et al. The growing burden of tuberculosis: global trends and interactions with the HIV epidemic. Arch Intern Med. 2003;163:1009-1021.

3. Sungkanuparph S., Manosuthi W., Kiertiburanakul S., et al. Initiation of antiretroviral therapy in advanced AIDS with active tuberculosis: clinical experiences from Thailand. J Infect. 2005; 1-7

4. Pozniak A.L., Miller R., Ormerod L.P. The treatment of tuberculosis in HIV-infected persons. AIDS. 1999;13:435-445.
5. Aaron L., Saadoun D., Calatroni I., et al. O. Tuberculosis in HIVinfected patients: a comprehensive review. Clin. Microbiol Infect. 2004; 10: 388-98.

6. Schmitt C, Riek M, Winters K, Schutz M et al. Unexpected hepatotoxicity of rifampicin and Saquinavir/Ritonavir in Healthy Male Volunteers. Arch Drug Info 2009;2:8-16

7. Pedral-Sampaio D.B., Alves C.R., Neto E.M., et al. Efficacy and Safety Efavirenz in HIV Patients on Rifampicin for Tuberculosis. Braz. J. Inf. Dis. 2004, 8:211-216.

8. Brasil, Ministério da Saúde. Guia de Tratamento: Recomendações para Terapia Anti-retroviral em Adultos e Adolescentes Infectados pelo HIV: 2008. Editora MS, Brasília. 2008. (disponível em www.aids.gov.br)

9. Collins K.R., Quinones-Mateu M.E., Toossi Z., et al. Impact of tuberculosis on HIV-1 replication, diversity, and disease progression. AIDS Rev. 2002, 4:165-76.

10. Rolla V., Vieira M.A., Pinto D., et al. Safety efficacy and Pharmacokinetics of Ritonavir 400mg and saquinavir400mg twice daily plus rifampicin combined therapy in HIV patients with tuberculosis. Clin Drug Invest. 2006;26(8):469-479.

11. López-Cortés LF, Ruiz-Valderas R, Viciana P, et al. Pharmacocinetic interactions between Efavirenz and RIFAMPICIN in HIV-infected patients with tuberculosis. Clin Pharmacokinet 2002;41:681-90.

12. Torres, T. S. and Rolla, V. C. What is the best strategy for treating TB-HIV co-infected patients with HAART and rifampicin without saquinavir? Int J Tuberc Lung Dis 2007;11(8):993-94

13. Saraceni V, King BS, Cavalcante SC, et al. Tuberculosis as primary cause of death among AIDS cases in Rio de Janeiro, Brazil. Int J Tuberc Lung Dis. 2008 Jul; 12(7):769-72.

14. Egger M, May M, Chene G, et al. Prognosis of HIV-1-infected patients starting highly active antiretroviral therapy: a collaborative analysis of prospective studies. Lancet. 2002; 360:119-29.

15. Campos DP, Ribeiro SR, Grinsztejn B, et al. Survival of AIDS patients using two case definitions, Rio de Janeiro, Brazil, 19862003. Aids 2005,19 Suppl 4:S22-26.

16. Boesecke C, Cooper DA. Toxicity of HIV protease inhibitors: clinical considerations. Curr Opin HIV AIDS. 2008 Nov;3(6):653-9

17. Moreno S, Hernández B, Dronda F. Antiretroviral therapy in AIDS patients with tuberculosis AIDS Rev. 2006 Jul-Sep;8(3):115-24.

18. Karim SA, Naidoo KJ, Glober A, et al. Initiating ART during TB Treatment Significantly Increases Survival: Results of a Randomized Controlled Clinical Trial in TB/HIV-co-infected Patients in South Africa [abstract 36a]. In. Program and abstracts: 16th Conference on Retroviruses and Opportunistic Infections (Montreal) Canada 2009. 